\title{
Vínculo social e individualización: reflexiones en torno \\ a las posibilidades del aprender
}

\author{
Margarita Palacios y Ana Cárdenas ${ }^{1}$
}

\section{Resumen}

A partir de la discusión de los conceptos de vínculo social e individualización, junto con el análisis de las principales transformaciones ocurridas en el mundo de la familia y del trabajo, este artículo ofrece un marco interpretativo para la comprensión del fenómeno del bajo rendimiento escolar de los alumnos en Chile. Dado que la individualización se caracteriza por el fraccionamiento y relativización de horizontes simbólicos y por el creciente desprendimiento inter-subjetivo, se propone en este artículo que tales transformaciones afectan la "educabilidad" de los niños, en tanto las condiciones afectivas requeridas en el aprendizaje escolar, tales como la confianza, pertenencia y certeza, estarían siendo paulatinamente debilitadas en nuestra sociedad.

\section{Social Ties and Individualization: A discussion about the possibilities of school learning}

\begin{abstract}
The concepts of social ties and individualization shed light in this article to a discussion about how the main transformations that have taken place in the institutions of the family and work are related to the phenomenon of low school performance in Chile. More specifically, it is argued that the fractioning of symbolic horizons and the increasing weakening of social ties that are associated to the process of individualization, hinder the very "educability" of children, insofar the affective conditions required in learning, such as trust, belonging and certitude, are increasingly weakened in our society.
\end{abstract}

\footnotetext{
${ }^{1}$ Margarita Palacios, Doctora en Sociología New School for Social Research, Nueva York. Profesora Instituto Estudios Latinoamericanos, Universidad Libre de Berlín e Investigadora asociada al Instituto de Ciencias Sociales de la Universidad Diego Portales, Santiago de Chile (mpalaciosb@yahoo.com).Ana Cárdenas, MA Universidad de Heidelberg, Doctora C en Sociología, Universidad Libre de Berlín (actomazic@hotmail.com).
} 


\section{Introducción}

El rendimiento escolar ha sido reconocido por la literatura especializada como el producto complejo de la interacción entre entorno familiar y escuela (Brunner y Elaccqua 2002). Diversos enfoques dan primacía a uno u otro, o a componentes particulares dentro de uno y de otro. Es así que factores tales como el origen socioeconómico del alumno, la educación y ocupación de los padres, los recursos del hogar y el nivel educacional de la comunidad, se acompañan de otros factores, en los que se subraya la importancia del nivel de involucramiento de los padres en la educación de sus hijos, el liderazgo, la confianza interpersonal, el nivel de asociatividad, el clima escolar y por supuesto, las creencias y los valores asociados a la educación. Diversas constelaciones de éstas variables pueden tanto colaborar como dificultar la educabilidad ${ }^{2}$ de niños y jóvenes.

Otros factores no relacionados directamente con familias y escuelas específicas, sino que con la sociedad en su conjunto, también afectan el cómo ocurre el proceso del aprendizaje. Es así que la globalización, la innovación tecnológica, la flexibilidad, la alta competitividad y su consecuente cambio en la organización laboral, han presentado también nuevos retos al sistema educacional. La velocidad en la que se desarrollan nuevos conocimientos y la posibilidad de acceder a inmensos volúmenes de información hace necesaria la educación permanente de los sujetos, de modo de que éstos no queden rápidamente descalificados y por ende, fuera del mercado laboral.Tal como afirma Juan Carlos Tedesco,"aprender a aprender y aprender a vivir juntos han sido postulados como los dos pilares que expresan los nuevos desafios que debe enfrentar la educación en el marco de las profundas transformaciones que vive la sociedad. El primero de ellos sintetiza los desafios educativos desde el punto de vista del desarrollo cognitivo, mientras el segundo sintetiza los desafios relativos a la construcción de un orden social donde podamos convivir cohesionados pero manteniendo nuestra identidad como diferentes" (Tedesco 2003:1). Estas dos dimensiones, cognitiva y afectivo-normativa (del saber "vivir juntos") y tal como veremos de aquí en adelante, están también tremendamente inter-penetradas, por lo que el éxito del proceso educativo depende de la simultaneidad de ambas.

En nuestro país la modificación educativa más significativa en estas últimas décadas ha sido la expansión de la oferta educacional, la que ha alcanzado casi una universalización de la educación básica ${ }^{3}$. En términos de su distribución al interior de los ${ }^{2}$ Educabilidad definida como "una construcción social que trasciende al sujeto y su familia, y que da cuenta del desarrollo cognitivo básico que se produce en los primeros años de vida - vinculada a una adecuada estimulación afectiva, buena alimentación y salud - y la socialización primaria mediante la cual los niños adquieren los rudimentos de un marco básico que les permite incorporarse a una situación especializada distinta de la familia, como es la escuela. La idea central es que todo niño nace potencialmente educable, pero que el contexto social opera, en muchos casos, como obstáculo que impide el desarrollo de esta potencialidad", ver Tedesco y Lopez (2002).

${ }^{3}$ De acuerdo a las estadísticas oficiales del Ministerio de Educación de Chile, la cobertura de atención por niveles de enseñanza creció en el período 1990-2001 desde un 95\% a un 97\% en el caso de la enseñanza básica y desde un 77\% 
diversos niveles socioeconómicos, se puede observar que se ha producido un aumento de la escolaridad en todos los deciles de la población, pero con mayor fuerza en los sectores más pobres (Teitelboim y Salfate 2003) ${ }^{4}$. Sin embargo, dichos resultados cuantitativos contrastan con los resultados cualitativos relativos al bajo rendimiento escolar (MINEDUC 2005). Esta situación paradójica de la educación, caracterizada por la simultaneidad de la extensión prácticamente universal de su cobertura junto con el bajo rendimiento escolar de los alumnos, hace necesario enfocarse en variables de tipo socio-culturales que si bien no son enteramente independientes de las variables económicas, si gozan de su propia lógica y sentido (Alexander 2003, Geertz 1973).

De este modo, aunque la relación rendimiento/grupo socioeconómico del alumno quede enteramente demostrada al analizar los resultados de la prueba Simce por tipo de establecimiento educacional (donde los colegios particulares de nivel socio económico medio y alto obtienen considerablemente mejores puntajes que la mayoría de los establecimientos públicos), las variables de entorno son particularmente interesantes para entender las diferencias dentro de cada grupo y, en particular, dentro de los establecimientos públicos. En efecto, estudios cualitativos realizados a nivel internacional (Machado 2002) en escuelas exitosas de escasos recursos dan cuenta precisamente de la buena calidad del tejido social alrededor de éstas, donde destaca la participación activa de los estudiantes y los apoderados, así como también el trabajo en equipo entre los profesores y directores de escuela.

Desde esta perspectiva cualitativa, que privilegia la subjetividad que acompaña los procesos sociales en general y el proceso educativo en particular, quisiéramos contribuir al debate respecto de la educación en Chile y vincularlo con procesos de transformación "simbólica" al interior de nuestra sociedad. Sin embargo, más que enfocarnos en variables de entorno propias de cada establecimiento educacional, nuestra perspectiva privilegia el análisis del proceso general de "individualización" que atraviesa nuestro país y el consecuente debilitamiento del vínculo social que este proceso conlleva. Siguiendo con las nociones expuestas anteriormente, se podría decir que la dimensión cognitiva del aprendizaje escolar se ha visto afectada (en este caso perjudicialmente), por las condiciones afectivo-normativas imperantes hoy en día, caracterizadas por el fraccionamiento y relativización de horizontes simbólicos colectivos, la creciente autonomía y la falta de participación de los individuos en tareas colectivas. Según nuestro parecer, el bajo rendimiento escolar de los alumnos de escuelas públicas en Chile está relacionado precisamente con un debilitamiento del vínculo social en nuestro país.

a un $85 \%$ en el caso de la enseñanza media (MINEDUC 2002). Cabe destacar que esta casi universalización de la educación básica se observa en todas las regiones del país (Teitelboim y Salfate 2003)

${ }^{4}$ Los resultados de expansión del sistema educacional chileno pareciera ser significativo no sólo en términos de la realidad nacional, sino también al interior del continente latinoamericano. Por ejemplo, Chile presenta los más altos porcentajes de nivel educacional secundario respecto a los países del MERCOSUR.A su vez, las tasas de abandono del sistema escolar por nivel de enseñanza son las más bajas dentro de esa zona geográfica (MINEDUC 2001). 
Este es un fenómeno que no es fácil de comprender, primero, porque no es cuantificable. Segundo, porque tiene un carácter paradójico y multidimensional. Tal como lo veremos más adelante, el proceso de individualización si bien favorece una creciente autonomía de los individuos y una mayor porosidad en la vida social - donde pluralidad de estilos de vida y el multiculturalismo aparecen como ideales sociales - éste también debilita el vínculo social y el sentido de pertenencia entre los individuos, dificultando de este modo la "educabilidad" de los niños y jóvenes.

Como es de esperar, si bien el debilitamiento del vínculo social relacionado con el proceso de individualización afecta a la población en su conjunto, en países con mayor desigualdad social su impacto "desintegrador" es más evidente ${ }^{5}$. Es así que en América Latina no sólo ha aumentado considerablemente la violencia juvenil ${ }^{6}$ (y la apatía política de este segmento social7), sino que los grupos socio-económicos más pobres, al "acumular riesgos" (psicológicos, socioeconómicos y culturales), enfrentan las mayores dificultades para enfrentar de un modo exitoso la educación (Bravo 2002).

Para el desarrollo en profundidad de este argumento, presentamos en las secciones que siguen los conceptos de vínculo social y de individualización, a la vez que analizamos cómo las transformaciones en las instituciones de la familia y del trabajo no sólo han alterado los modos tradicionales de socialización, sino que dan muestras también de claras tendencias de desprendimiento intersubjetivo. Si bien aún no contamos con la evidencia empírica que avale cada una de éstas afirmaciones, nuestro objetivo por ahora es presentar el marco teórico que estimamos puede contribuir en la elaboración de un marco interpretativo que amplíe el foco para la comprensión de este fenómeno.

\section{Socialización, vínculo social y aprendizaje escolar}

La presencia del vínculo social es una de las condiciones básicas en el proceso del aprendizaje. A nivel de los alumnos, este vínculo se traduce en una identificación primaria (Freud 1959) ${ }^{8}$ del niño con su entorno más inmediato (vínculo básico afec-

\footnotetext{
${ }^{5}$ Cabe mencionar aquí que para el caso de América Latina, el proceso de individualización adquiere dimensiones aún más complejas, en tanto la región - a diferencia de Europa y Norteamérica - se ha caracterizado históricamente por su alta desigualdad social y también por sus prolongadas experiencias de autoritarismo y violencia. Esto es, el proceso de debilitamiento del vínculo social ocurre en sociedades ya altamente fragmentadas y muchas veces con instituciones que no gozan de gran legitimidad dentro de la población.

${ }^{6}$ Según el Informe de Paz Ciudadana, durante el año 2000 54\% de los robos con violencia fue protagonizado por menores de 19 años y entre los años 1995 y 2000, los jóvenes entre 14 y 18 años aprehendidos por este tipo de delito aumentaron en un $410 \%$ (www.pazciudadana.cl/diagnostico).

${ }^{7}$ En el sistema electoral chileno la inscripción electoral es voluntaria, pero la votación es obligatoria. En este contexto, la inscripción de los jóvenes en los registros electorales desde el año 1988 ha venido presentando una baja sistemática, ubicándose ésta en un 16.4\% en las últimas elecciones presidenciales 99/2000 (www.electoral.cl/estudios).

${ }^{8} \mathrm{El}$ concepto de identificación es fundamental en la teoría psicoanalítica. Este no sólo da cuenta de la exterioridad (o dependencia del sujeto con respecto del "otro" con el cual se identifica), sino que también describe el carácter "libidinal" de la identidad, es decir el sujeto no sólo "es alguien", sino que tiene una "pasión de ser". La identificación es descrita por Freud como el primer vínculo afectivo del sujeto, en la que uno de los padres juega el rol de modelo (o referente) sexual,
} 
tivo con el padre-madre o quien lo cuida), la que permite tanto la generalización del aprendizaje (Ausubel 1976) ${ }^{9}$ como la confianza básica en un mundo "incorporable" (Winnicott 1993) y no amenazante. El vínculo social provee a los adultos involucrados en el proceso educativo, por otra parte, de fe en los alumnos, en la educación y en ellos mismos en sus roles respectivos (Blumer 1969) ${ }^{10}$. Puesto que el aprendizaje requiere de la entrega del "yo" al "otro", la presencia de este vínculo es fundamental para poder encarnar los roles asociados a la educación. Tanto los roles de alumno, profesor y apoderado requieren de una creencia específica relacionada con la fe en el otro y en sí mismo. Sólo de ese modo se valora (o al menos se soporta) la postergación de la satisfacción inmediata y es posible someterse al esfuerzo y la disciplina que es aprender y enseñar respectivamente.

El aprendizaje escolar ciertamente es parte de un proceso más general de socialización. Éste se caracteriza por la adquisición de los referentes simbólico-culturales de la comunidad a la que se incorpora el sujeto, la capacidad de éste de ponerse en la situación del otro y la posibilidad de diálogo del sujeto consigo mismo y con el resto de la sociedad (Alexander 1987, Giddens y Turner 1987). Este proceso de socialización descrito como de identificación simbólica y que conduce a la integración social ${ }^{11}$, al igual que el proceso específico del aprendizaje escolar, requiere de ciertas condiciones específicas relacionadas con la existencia de un universo simbólico con el cual el sujeto se identifica y de ciertas condiciones específicas relacionadas con la propia vida del individuo. En este caso nos referimos básicamente a la existencia de un "nexo" o instituciones intermedias (tal como la familia, el trabajo y la escuela) que garanticen no sólo la transmisión cultural, sino que además provean al sujeto de las condiciones normativas, cognitivas y afectivas que éste requiere para el desarrollo de su sociabilidad y autonomía (Honneth 1996). La incorporación de los referentes simbólicos no sólo proveen entonces al sujeto del lenguaje que permite su incorporación a la vida comunitaria (compartiendo el sentido común y proyectos de ésta), sino que a la vez orientan y dan sentido a la vida del sujeto: se establece un horizonte, un "yo ideal", y se traza el camino para alcanzarlo.

\footnotetext{
mientras que el otro es "investido sexualmente" como objeto del deseo.

${ }^{9}$ La generalización del aprendizaje desde un punto de vista cognitivo (y no conductista) se da cuando hay aprendizaje de "buena calidad", es decir cuando existe "comprensión" y no repetición mecánica.

${ }^{10} \mathrm{La}$ orientación de la acción está directamente relacionada con la interpretación que se hace de ella, por lo que el "sentido" o la alta valoración (en este caso de la educación), garantizan el buen desempeño de cada una de las funciones asociadas a la educación.

${ }^{11} \mathrm{La}$ integración social no se refiere a la igualdad social en términos económicos o sociales, sino a la presencia de un vínculo cultural-moral básico que une al individuo al resto de la sociedad. Este vínculo, o "ethos social", para ser efectivo necesita trascender la voluntad, la racionalidad y los criterios estratégicos de los individuos. Es decir, este vínculo opera como un mandato "sagrado" que sienta las bases, orienta y legitima la vida en comunidad. (Ver Durkheim 1984).
} 


\section{El Concepto de individualización}

Cambios tanto en el universo simbólico, descrito como cada vez más fraccionado y débil (Bell 1999, Kumar 1995), como en las redes y organizaciones socializadoras, han alterado, sin embargo, este modo tradicional de socialización. El debilitamiento o desaparición de instituciones intermedias, junto con la creciente autonomía y la falta de participación de los individuos en tareas colectivas, han sido descritas en la sociología europea y americana como un proceso de "individualización" (Beck, Giddens y Lash 1994, Beck y Beck-Gernsheim 2002, Giddens 1991, Inglehart 1990, Lasch 1991, Sennett 1998, 2006, Bauman, 2001, 2006).

La teoría de la individualización ${ }^{12}$ describe una paulatina disolución y desprendimiento de las formas de vida propias de la sociedad industrial (tales como la clase, la familia y el orden tradicional de género), producto principalmente de un mejoramiento en las condiciones materiales de vida, la expansión del sistema educacional, el aumento del tiempo libre y la movilidad geográfica en las sociedades europeas, específicamente alemana (Beck 1986, Beck-Gernsheim 1994, Beck y Beck-Gernsheim 1993, 1994) ${ }^{13}$. En dicho contexto, se estaría produciendo un debilitamiento del contenido normativo de dichas instituciones y, consecuentemente, una expansión de las posibilidades de elección y decisión de los sujetos.

Sin embargo, dicha teoría plantea que los individuos perderían paralela y crecientemente parte de las seguridades y certezas que dichas instituciones les habían proporcionado en el pasado ${ }^{14}$, quedando entonces sin su "hogar del ser" (die Heimat des Ichs) (Berger, Berger y Kellner 1975). Los sujetos tendrían entonces que ir asumiendo paulatinamente las responsabilidades y posibilidades, los riesgos e (in)seguridades relativos a la definición, planificación y organización de su propia forma de vida y de sus relaciones sociales (Beck 1986, Beck-Gernsheim 1994, Beck y Beck-Gernsheim 1993, 1994). Consecuentemente, los sujetos ya no podrían cumplir con la tradicional "trayectoria de vida" que socialmente caracterizó a las sociedades industriales (Kohli, 1985, 1986) y bajo las cuales se estructuraron las instituciones básicas de socialización (por ejemplo, la escuela), debiendo cada individuo definir y organizar su propia biografia ${ }^{15}$. Estas tenderían entonces a tener un horizonte de carácter "abierto" (Kohli 1989, Böhnisch 2001), volviendo así altamente "riesgosas" (Beck y Beck-Gernsheim 1994) y consecuentemente inciertas tanto las posibilidades de desarrollo de los sujetos como de la sociedad.

\footnotetext{
${ }^{12} \mathrm{Al}$ interior de la sociología se han desarrollado tanto una teoría "negativa", como "positiva" y "ambivalente" respecto al así llamado "proceso de individualización”. El nuevo impulso que tendría dicho proceso corresponde principalmente al tipo "ambivalente" de esta teoría. Al respecto ver Schroer (2000).

${ }^{13}$ Las críticas relativas al actual desarrollo de dicha teoría apuntan a la falta de evidencias empíricas (Burkart 1993) y a la ambigüedad de sus postulados (Kron 2000).

${ }^{14}$ Como por ejemplo, la posibilidad de movilidad social "asegurada" por el origen social de la persona.

${ }^{15}$ Diversos autores han tematizado dicho proceso en términos de biografias a elección (Ley 1984), biografias reflexivas (Giddens 1991), biografias autoreflexivas (Beck 1986) y biografias creables (Beck y Beck-Gernsheim, 1993, Hitzler y Honer 1994) (los conceptos mencionados han sido traducidas por las propias autoras desde el idioma original).
} 
Por último, cabe tener presente que dicho proceso de individualización adquiere características muy específicas, arraigadas en el propio proceso de desarrollo que han tenido las sociedades modernas. Es así como el proceso de desprendimiento de los individuos, de los vínculos sociales tradicionales y su consecuente diferenciación trae consigo una mayor dependencia del Estado y del Mercado (laboral), es decir, de organizaciones e instituciones impersonales (Beck 1986, Beck y Sopp 1997). Dicho proceso tiende entonces a generar nuevas formas de estandarización y dependencia institucional hacia los sujetos (ibid.).

\section{La individualización en Chile}

A pesar de que al interior de la sociedad chilena no se ha alcanzado el nivel de bienestar material y de seguridad social que habría favorecido el proceso de individualización al interior de las sociedades europeas (y norteamericana), la literatura actual da cuenta de una paulatina disolución y un desprendimiento de las formas de vida que han imperado en la sociedad chilena tales como la precarización de las condiciones de trabajo, junto a una transformación y generación de nuevos tipos de familias, una creciente incorporación de las mujeres al mercado del trabajo y la conformación de diversos modos de vida al interior de la población (PNUD 2000, 2002, INE 2003). En lo que sigue describimos algunos de estas transformaciones, las que a nuestro parecer, dan cuenta del proceso de debilitamiento del vínculo social al interior de nuestro país y que indirectamente afectan la dimensión "afectivo-normativa" necesaria para el aprendizaje escolar.

\section{IV.1. Empleo}

Uno de los principales cambios ocurridos en Chile en el contexto de la liberalización de la economía durante las décadas de los setentas y ochentas se refiere a las reformas al Código del Trabajo ${ }^{16}$. Estas buscaron flexibilizar el factor trabajo, sus costos, las relaciones colectivas (Tokman y Martínez 1999) y las relaciones individuales de trabajo ${ }^{17}$. Estas últimas (materializadas como reformas del contrato de trabajo), contemplaron tanto las condiciones de contratación así como la duración de los contratos, las condiciones de despido y los costos de contratación. A partir de estas reformas surgieron nuevas modalidades de contratación temporal ${ }^{18}$, las que no sólo ${ }^{16} \mathrm{El}$ proceso de reforma al Código del Trabajo en Chile comprendió tanto la modificación del contenido de las normas laborales ("re-regulación" o "adaptación”) como la supresión de algunas de sus normas (“deregulación”). (Ver Morgado-Valenzuela 1999 y Urmeneta 1999).

${ }^{17}$ Cabe tener presente que el Plan Laboral, específicamente, el Decreto ley 2.200 (1978) y la Ley 18.018 (1981) introdujeron reformas relativas tanto a las relaciones individuales como colectivas de trabajo (Tokman y Martínez 1999, Urmeneta, 1999).

${ }^{18}$ Estas comprenden la contratación permanente (indefinida) y la contratación no permanente (definida), dividiéndose esta última en modalidades de contrato a plazo fijo y duración temporal (por temporada, por obra o trato y por servicios). 
han tenido efectos desestabilizadores para la vida de los trabajadores, sino que éstas también han modificado y debilitado el contenido normativo de una de las instituciones centrales en torno a las cuales se había organizado la vida social en Chile: el "trabajo típico"19.

La transformación de las condiciones de trabajo en Chile ha sido interpretada como un proceso creciente de "precarizarización" de las condiciones de trabajo asalariado ${ }^{20}$, a través del incremento de puestos de trabajo por tiempo definido y/o no regulado legalmente y consecuentemente, con escasa o nula protección social. Más aún, se ha planteado que dicho proceso corresponde a un proceso de "exclusión social relativa" (Wormald y Ruiz Tagle 1999). A través de éste, la mayor parte de la población habría experimentado un proceso paulatino de "inclusión" al crecimiento económico, a través de las mayores posibilidades de empleo y de ingresos. Sin embargo, dicho proceso estaría acompañado de un proceso paralelo de "exclusión" de parte de la fuerza de trabajo de las condiciones "típicas" de trabajo, situando especialmente a los/as trabajadores/as más pobres y a aquellos sectores de la economía con mayor presencia de trabajadoras mujeres (agricultura, comercio y servicios) en una situación de relativa mayor inestabilidad laboral y parcial o totalmente al margen de la seguridad social.

Estos enfoques de tipo "estructural" parecieran no lograr explicar en su totalidad la generación de empleos, socialmente desprotegidos e inestables, ocurrido en estas últimas décadas en Chile. Si se considera el análisis de la "subjetividad" que acompaña dichos procesos, se observa que la transformación de las condiciones de trabajo en Chile pareciera ser reforzada y profundizada por las propias opciones y acciones de los sujetos (Cárdenas, 2005, Martínez y Palacios 1996). Estos tenderían a privilegiar, por lo menos en parte, condiciones de trabajo más inestables y con escaso o nulo derecho a la seguridad social a cambio de poder alcanzar un mayor equilibrio en su integración a las diversas esferas de esta sociedad (por ejemplo, no sólo en el trabajo, sino también en la familia y/o la educación) y lograr alcanzar mayores grados de libertad y control sobre sus vidas, a pesar de los riesgos e inestabilidades sociales y laborales asociados a dichas formas de integración (Cárdenas 2005).

Es precisamente en el marco de dicho proceso de individualización donde el proceso de transformación de las condiciones de trabajo en Chile pareciera volverse especialmente "riesgoso" para, por lo menos, la fuerza de trabajo perteneciente a los grupos socioeconómicos más pobres. Al producirse un debilitamiento del contenido normativo de la institución "contrato de trabajo" los sujetos se verían forzados y, a su vez, estarían optando integrarse al mercado del trabajo bajo condiciones parcial o

\footnotetext{
${ }^{19}$ Elementos básicos del "trabajo típico" fueron la existencia de un contrato de duración indefinida, un solo empleador y un solo lugar de desempeño laboral, la prestación de un servicio continuo, un régimen de jornada completa de trabajo, una organización del trabajo rígida, derecho a la previsión social y protección legal de ciertos derechos autónomos o heterónomos, el derecho a la sindicalización y la negociación colectiva (Guerra 1994).

${ }^{20}$ Diversos estudios empíricos dan cuenta de dicho proceso.Ver Díaz, Medel y Schläen (1996), Reinecke (1997), Díaz y Yáñez (1998), Echeverría y Uribe (1998), OIT (1998), Wormald y Ruiz-Tagle (1999).
} 
totalmente inestables y desprotegidas legalmente ${ }^{21}$. De esta manera, serían los grupos socio-económicos más educados y consecuentemente de mayor bienestar económico en Chile los que podrían expandir sus posibilidades de elección y decisión respecto a sus condiciones de trabajo y, al mismo tiempo, asumir más fácilmente los riesgos e inestabilidades sociales y laborales asociados a dicha forma de integración (Cárdenas, 2005). Por el contrario, serían los grupos socio-económicos más pobres, los que a pesar de intentar optar parcial o totalmente por determinadas condiciones de trabajo, parecieran no contar con los recursos sociales, culturales y personales que permitirían compensar los riesgos asociados a tales opciones (ibid.).

Las transformaciones en el mundo del trabajo no sólo han afectado a las familias, sino que también a la sociedad en su conjunto. El trabajo ha sido una de las principales fuentes de sentido e integración al interior de las sociedades industriales (Castel 1996), es decir, una esfera social donde se produce un encuentro con un otro (en este caso, con otros/as trabajadores/as) y por lo tanto, donde los sujetos participan en una determinada "comunidad de trabajo". En este sentido, el contrato de trabajo indefinido ha asegurado y simbolizado la estabilidad de dicha comunidad, específicamente la permanencia de sus miembros al interior de ésta. Consecuentemente, el surgimiento y la proliferación de contratos de trabajo por tiempo definido, la total inexistencia de contratos de trabajo (el caso más extremo) así como las propias opciones y acciones de los sujetos parecieran estar debilitando dichos lazos simbólicos que institucionalmente habían logrado ser asegurados.

\section{IV.2. Familia}

La familia, una de las principales instituciones socializadoras de nuestra sociedad, no ha quedado al margen de las transformaciones sociales y culturales de las últimas décadas, y como tal, su análisis también ha sido considerado desde la teoría de la individualización (Beck 1986, Beck-Gernsheim 1994, 1994a, Beck y Beck-Gernsheim 1993). Dentro de este análisis se destaca fundamentalmente la transformación de la morfología de la familia, representada por ejemplo en el incremento de los divorcios y de los hogares unipersonales y monoparentales femeninos, el aumento de las convivencias y de las familias recompuestas, una disminución de la tasa de natalidad y un incremento de los nacimientos fuera del matrimonio, y la postergación de la maternidad/paternidad a edades más tardías.

Estas mismas tendencias son posibles de ser observadas al interior de la sociedad chilena. Si bien los hogares nucleares ${ }^{22}$ con hijos/as continúa siendo la estructura ${ }^{21}$ Diversos estudios han venido dando cuenta del desarrollo de "trayectorias laborales orientadas hacia el cambio" (Mauro, Godoy y Guzmán 2001), donde la inestabilidad laboral es una de los ejes centrales respecto al cual se han venido van desarrollando las trayectorias laborales de los trabajadores/as en estas últimas décadas (Guzmán, Mauro y Araujo 1999, Mauro, Godoy y Muñoz 2001, Espinoza y Morris 2002, Dirección del Trabajo 2003, Guzmán y Mauro 2004, 2004a).

${ }^{22}$ Datos relativos a los dos últimos Censos de Personas y Hogares realizados en 1992 y el año 2002, respectivamente. 
predominante en Chile (47.8\%), es posible observar que las formas de constitución de las familias han tendido a diversificarse en estas últimas décadas y a desinstitucionalizarse respecto del patrón de la llamada "familia industrial": el tamaño de los hogares ha venido reduciéndose $\mathrm{e}^{23}$, se ha producido una disminución de los hogares formados por ambos padres e hijos/as y un aumento de los hogares monoparentales (principalmente encabezados por mujeres) ${ }^{24}$ y nucleares biparentales sin hijos/ as (Gubbins, Browne y Bagnara 2003). A su vez, se ha producido un aumento de la convivencia ${ }^{25}$, las separaciones y el divorcio. Por último, hoy en día nacen más hijos fuera que dentro del matrimonio, superando incluso las tasas conocidas para el siglo XIX (Valdés 2007).

Si bien la familia chilena sigue siendo bastante tradicional (Palacios 2006, Martínez y Palacios 2005), los roles clásicos de padre proveedor y madre dueña de casa se han visto claramente desestabilizados, no sólo en términos de su cuestionamiento como referentes valóricos, sino que efectivamente ha habido un cambio en el comportamiento de la población, siendo su manifestación más significativa el aumento de la participación laboral femenina ${ }^{26}$, lo que ha redundado, por ejemplo, en un incremento de las familias con doble ingreso en un 40\% (Valdés 2007).

Aunque las transformaciones ocurridas dentro de la familia en nuestro país, coinciden en términos generales con las transformaciones de las sociedades europeas y norteamericana, el caso chileno presenta algunas particularidades que son especialmente significativas para nuestro estudio. El primer elemento a destacar es que Chile se ha destacado por su retraso en materias legislativas. En efecto, la aprobación de la Ley de Divorcio en este país es relativamente reciente (junto con Malta constituían las dos excepciones del mundo occidental), así como también sólo recientemente los hijos nacidos fuera del matrimonio adquirieron igualdad de derecho. Por otro lado, es necesario también afirmar que si bien la familia se ha diversificado, la "idea" de familia continúa siendo un referente simbólico fundamental en el imaginario de los individuos (Palacios 2006), quienes pese a sus variadas experiencias familiares, intentan construir narrativas familiares coherentes, y que les otorguen contención y sentido a sus vidas.

Esta distancia entre la experiencia y la "imaginación" ha producido que esta transición cultural conlleve altos grados de contradicción simbólica: el liberalismo (que acompaña la redefinición de género) no ha superado el previo conservadurismo, sino ${ }^{23} \mathrm{El}$ promedio de integrantes del hogar en 1992 era de 4 personas, mientras que en el año 2002 era de 3.6 personas (Gubbins, Browne y Bagnara, 2003). Cabe tener presente que la tasas de fecundidad se redujo entre 1960 y el año 2005 de 5,7 hijos a 2,2 hijos (Valdés 2007).

${ }^{24}$ De acuerdo a la Encuesta de Caracterización Socioeconómica (CASEN) del Ministerio de Planificación (MIDEPLAN), los hogares monoparentales con hijos tuvo un incremento de un 22.2\% a un 25.6\% en el período $1990-2005$. Esto ha significado un aumento en la separación de la residencia y la filiación al interior de la familia en Chile (Valdés 2007).

${ }^{25}$ Entre 1992 y 2002 esta forma de unión de parejas aumentó de 6.2\% a 9.7\% (Gubbins, Browne y Bagnara 2003).

${ }^{26} \mathrm{La}$ tasa de participación femenina en zonas urbanas aumentó en el período 1990-2000 desde un 35.0 a un 42.0 (Abramo y Valenzuela 2006). 
que simplemente lo ha fragmentado. Tal como lo describimos en un trabajo anterior (Martínez y Palacios 2005), en Chile coexisten en los mismos sujetos y en relación a los mismos temas tanto un discurso conservador, basado en una "ideología conservadora" con claras raíces religiosas, y un discurso secular, que a diferencia del primero, no refleja una "ideología liberal" sino que simplemente una respuesta práctica a las demandas de la vida moderna. En nuestro reciente estudio acerca de las transformaciones en la familia chilena (Valdés, Castelain-Mounier y Palacios 2006) quedó nuevamente de manifiesto cómo este desfase discursivo ocasiona una profunda culpa ${ }^{27}$, falta de legitimidad e identificación con el estilo de vida adoptado.

Tal como lo hemos venido afirmando a lo largo del artículo, todas estas transformaciones tienen un carácter ambivalente. Obviamente se pueden $-\mathrm{y}$ de hecho son - consideradas como favorables, en tanto aumentan la autonomía de los sujetos. En el contexto de la individualización, tanto hombres como mujeres tienen mayores espacios para crear y personificar su estilo de vida, sin las limitaciones normativas que hasta hace poco marcaban claras diferencias y una relación de subordinación entre los géneros. Ahora bien, desde la problemática que nos ocupa ahora, estas transformaciones han alterado los modos tradicionales de socialización, y por ahora al menos, estas muestran también efectos negativos para la constitución del vínculo social, fundamentalmente en tanto se desvanece la "certeza". Tal como lo afirma Ximena Valdés en la introducción a Puertas Adentro: Femenino y Masculino en la Sociedad Chilena, "reinventar la vida privada supone un cierto rediseño de la familia: el reacomodo de lugares, funciones y relaciones entre el hombre y la mujer, el padre y la madre y de ambos con respecto a los hijos en sintonía con lo que circula en la sociedad que reclama a hombres y mujeres en el espacio público. Como todo ensayo y tentativa por dejar atrás lo conocido, este tipo de búsqueda implica rupturas, produce desorden, a menudo genera incertidumbre, y también resistencias que llevan al acantonamiento de las personas en la tradición. Sin embargo, un proceso de transición de esta naturaleza deja un campo abierto a nuevas formas de vida que sin contar con las certezas del pasado, buscan distanciarse de las normas así como equilibrio y satisfacción en las relaciones inter-personales pero a la vez devienen más frágiles e inseguras" (Valdés, Castelain-Meunier y Palacios 2006: 8).

\section{IV.3. Nuevas condiciones de la socialización}

Considerando las transformaciones descritas en las secciones anteriores, en relación al mundo del trabajo y la redefinición de la vida privada, podría afirmarse que la actual generación de padres trabajadores no sólo enfrenta dificultades para configurar un horizonte común para sí y con otros, sino que también en la transmisión de los referentes simbólico-culturales básicos a sus hijos/as. Según los resultados de nuestro

${ }^{27}$ Sentimiento expresado fundamentalmente por mujeres de distintos estratos sociales que trabajan fuera del hogar. 
estudio cuantitativo y cualitativo realizado durante 2007 en Santiago ${ }^{28}$, son los padres (y madres) con trabajos temporalmente definidos y/o al margen de la regulación laboral, pertenecientes a los grupos socio-económicos más pobres, quienes manifiestan una especial dificultad para poder transmitir un horizonte simbólico "confiable" y "no amenazante" a sus hijos, debido precisamente a la inestabilidad e incertidumbre asociada a este tipo de condiciones de trabajo y de vida en general. Cabe mencionar además aquí que dentro de este segmento social y especialmente en tramos de edad de "adulto-joven", la extrema informalidad económica tiende a asociarse con una "precariedad inter-subjetiva" o una improvisación permanente, en el sentido de que las relaciones afectivas (al menos con las parejas y los amigos), tienden a tener un carácter extremadamente circunstancial y muchas veces marcadas por experiencias de violencia o muerte.

Considerando lo anterior, la socialización "desde la certeza simbólica" de hijos de madres trabajadoras jefas de hogar, correspondientes a los sectores socioeconómicos más pobres, pareciera ser aún más problemática, debido a la relativa baja participación laboral de este segmento de la población ${ }^{29}$ (Abramo 2006). Esto nos lleva a suponer que las nuevas generaciones que participan como primera generación en el mercado del trabajo tendrían un horizonte simbólico aún más difuso y consecuentemente, dificilmente transferible a sus hijos. El incremento de los hogares monoparentales encabezados por una jefa de hogar otorga a este segmento poblacional, por las razones previamente descritas, una significación cada vez mayor en este proceso de transformación y debilitamiento del vínculo social.

En nuestro estudio "Los Nuevos Trabajadores de Chile" pudimos constatar también que se suma como factor de riesgo la larga jornada de trabajo a las cuales se ven sometidas una gran parte de la fuerza de trabajo más pobre, en la medida en que los padres/apoderados no sólo enfrentan dificultades para transmitir un horizonte simbólico común e ideal a sus hijos/as, sino que tampoco cuentan con el suficiente espacio temporal para reconstruirlo y transmitirlo.

En conclusión, para el caso de Chile cabe entonces pensar que la precariedad tanto laboral como de la constitución de los vínculos al interior de la familia, especialmente en el segmento más pobre de la población, se relacionan hoy en día con una cierta "precariedad simbólica", en tanto las posibilidades de la conformación (y experiencia) de horizontes simbólicos comunes y de vínculos sociales más sólidos, se vuelven cada vez más inciertas ${ }^{30}$.

\footnotetext{
${ }^{28}$ "Los Nuevos Trabajadores de Chile", financiado por Fundación Ford y OIT. Éste comprendió la realización de 8 Grupos Focales con Jóvenes estrato C3-D-E entre 18 y 24 años, 8 entrevistas en profundidad a 8 padres y madres (tanto trabajadores asalariados como informales) de los mismos estratos, y una encuesta a jóvenes realizada en Santiago.

${ }^{29} \mathrm{La}$ tasa de participación femenina correspondiente a los hogares de bajos ingresos en zonas urbanas creció desde un 21.0 en 1990 a un 29.0 en el año 2000 (Abramo y Valenzuela 2006).

${ }^{30} \mathrm{Un}$ ejemplo interesante al respecto es el caso de los/as trabajadores/as no calificados/as que forman parte de los últimos eslabones de las cadenas productivas surgidas en el marco del proceso de reorganización de las empresas en Chile. En la práctica, éstas presentan diversas formas de encadenamiento, donde la ubicación de los/as trabajadores/ as en los eslabones productivos puede volverse altamente compleja (Reinecke 1997). Consecuentemente, estos/as trabajadores/as tendrían escaso o nulo contacto con los/as trabajadores/as de la empresa "madre" y/o de los diferentes
} 


\section{Conclusiones}

A partir del análisis de las principales transformaciones de la familia y del mundo del trabajo, y utilizando los conceptos de vínculo social e individualización, este artículo ha buscado ampliar el foco de comprensión del fenómeno del bajo rendimiento escolar de los alumnos en Chile. El argumento presentado es que las transformaciones ocurridas al interior de nuestra sociedad han conllevado el debilitamiento del vínculo social, por lo que las condiciones "simbólicas" - tales como la confianza, pertenencia y la certeza - requeridas en el aprendizaje escolar, se han visto particularmente afectadas.

En particular, hemos enfatizado el carácter intergeneracional de este fenómeno, el que no se manifiesta únicamente a nivel de la población adulta (por ejemplo en el ámbito del trabajo y la familia), sino que también en el segmento más joven de la población (el que se manifiesta fundamentalmente en la escuela). La proliferación de diversas condiciones de trabajo y formas de constitución de la familia presenta crecientes desafios y dificultades a los padres/apoderados para socializar a las generaciones más jóvenes de la población en el marco de un horizonte simbólico común, puesto que los propios padres/apoderados carecen ellos mismos de dicho horizonte. Consecuentemente, tanto padres/apoderados como hijos/pupilos se ven obligados a construir sus propias biografias, con escasos referentes que orienten dicha construcción. Cabe no olvidar aquí al propio profesorado, quien no ha quedado fuera de éste proceso de transformación.

Podría afirmarse entonces que en el caso de los/as alumnos/as, la construcción de sus biografias ocurre con un "yo ideal" debilitado, restándole capacidad de orientación y sentido a la educación. Por otra parte, el propio mundo del trabajo, por las razones previamente explicadas, pareciera también restarle legitimidad a la escuela como institución. ${ }^{31}$ De este modo, el aprendizaje escolar está ocurriendo, especialmente en los sectores socioeconómicos más pobres, en el marco de instituciones sociales intermedias simbólicamente debilitadas, que no logran garantizar el proceso de identificación simbólica y consecuentemente, de constitución del vínculo social requerido para asegurar el aprendizaje escolar ${ }^{32}$.

Para terminar, nos gustaría "matizar" dos aspectos de nuestro argumento: En nuestro interés por comprender el fenómeno del bajo rendimiento escolar a la luz de las principales transformaciones de la familia y del trabajo, hemos asumido una lectura más bien crítica (casi normativa) respecto del proceso de individualización. Sin embargo,

eslabones productivos, pues generalmente realizan trabajos desde su domicilio o en pequeños talleres.

${ }^{31}$ Es necesario además agregar que hoy en día, el desempleo es un problema que afecta principalmente a este grupo poblacional. Según cifras provenientes de la Encuesta Nacional del Empleo, la tasa de desocupación a nivel nacional durante el período 2003-2004 se ha mantenido alrededor del 20,0 en el segmento etario 15-24 años (INE 2005).

${ }^{32}$ Ciertamente los jóvenes también cuentan con nuevos referentes simbólicos asociados tanto a los medios de comunicación como a sus propias biografías. 
nos parece de vital importancia no olvidar el carácter ambivalente, abierto y paradójico de este proceso de transformación social. Este no es el lugar para listar todos los "beneficios" asociados al proceso de individualización, por lo que sólo afirmaremos que evidentemente éste también conlleva una creciente democratización de la vida social, en tanto que la emergencia de nuevos estilos de vida, el multi-culturalismo y la creciente igualdad de género, le son también inherentes a este proceso.

Por otra parte, si bien formas tradicionales de integración social parecieran estar desapareciendo, nuevas formas de socialización emergen paralelamente. En este sentido, consideramos de vital importancia indagar respecto de nuevos referentes colectivos tales como los (nuevos) medios de comunicación, el barrio, las pandillas y la recreación, en tanto éstos no sólo constituyen nuevas fuentes de significado y pertenencia centrales en el mundo de los jóvenes, sino que evidentemente éstas también afectan el proceso del aprendizaje escolar. 


\section{Bibliografía}

Abramo, L. y Valenzuela, M.E. (2006): "Inserción laboral y brechas de equidad de género en América Latina" en: Abramo, L. (Ed.). Trabajo decente y equidad de género en América atina. Oficina Internacional del Trabajo (p.29-p.62).

Administradora de Fondos de Cesantía (2005): “Informe 2004" en: Economía y Negocios, diario "El Mercurio", Santiago de Chile (17.8.2005).

Alexander, J. (1987): Twenty Lectures, Columbia University Press.

Alexander, J. (2003): The Meanings of Social Life: A Cultural Sociology. New York: Oxford University Press

Arellano, J.P. (1985): Políticas sociales y desarrollo en Chile 1924-1984, CIEPLAN, Santiago de Chile.

Arellano, J.P (2004): "Políticas sociales para el crecimiento con equidad. Chile 19902002”, Serie Estudios Socio/Económicos, N²6, CIEPLAN, Santiago de Chile.

Ausubel, D. (1976): Psicología Educativa desde un punto de vista cognitivo, México:Trillas.

Beck, U. (1986): Risikogesellschaft. Auf den Weg in eine andere Moderne. Suhrkamp, Frankfurt.

Beck-Gernsheim, E. (1994):"“Individualisierungstheorie:Veränderungen des Lebenslaufs in der Moderne" en: Keupp, H. (ed.), Zugänge zum Subjekt: Perspektiven einer reflexiven Sozialpsychologie. Suhrkamp, Frankfurt am Main.

Beck-Gernsheim, E. (1994a): "Auf dem Weg in die postfamiliale Familie von der Notgemeinschaft zur Wahlverwandschaft" en: Beck/Beck-Gernsheim (1994) Riskante Freiheiten. Zur Individualisierung von Lebensformen in der Moderne. Suhrkamp, Frankfurt (p. 115-138).

Beck, U. y Beck-Gernsheim, E. (1993): "Nicht Autonomie, sondern Bastelbiographie. Anmerkungen zur Individualisierungsdiskussion am Beispiel des Aufsatzes von Günther

Burkart" en: Zeitschrift für Soziologie, Año 22, Cuaderno 3, F. Enke Verlag, Stuttgart (p. 178-187). 
Beck, U. y Beck-Gernsheim, E. (1994): "Individualisierung in modernen Gesellschaften- Perspektiven und Kontroversen einer subjektorientierten Soziologie". Riskante Freiheiten. Zur Individualisierung von Lebensformen in der Moderne. Suhrkamp, Frankfurt (p. 10-39).

Beck, U. y Sopp, P. (1997): “Einleitung: Individualisierung und Integration -Versuch einer Problemskizze” en: Beck, U. y Sopp, P. (Eds.), Individualisierung und Integration. Neue Konfliktlinien und neuer Integrationsmodus?, Leske + Budrich (p.9-19).

Bell, D. (1999): The Coming of Post-Industrial Societies: A Venture in Social Forecasting. Basic Books.

Berger, P., Berger, B. y Kellner, H. (1975): Das Unbehagen in der Modernität, Campus, Frankfurt.

Blumer, H. (1969): Symbolic Interactionism. Perspective and Method. Prentice Hall.

Böhnisch, L. (2001): Sozialpädagogik der Lebensalter. Eine Einführung. Juventa. Weinheim/München.

Bravo, L. (2002): Psicología de las dificultades del aprendizaje escolar. Santiago: Editorial Universitaria.

Brunner, J.J. y Elaccqua, (2002): Informe capital humano en Chile. Santiago: Universidad Adolfo Ibáñez.

Burkart, G. (1993): "Individualisierung und Elternschaft - Das Besipiel USA" en: Zeitschrift für Soziologie, Año 22, Cuaderno 3, F. Enke Verlag, Stuttgart (p. 159-177).

Burkart, G. (1993): “Eine Gesellschaft von nicht-autonomen biographischen Bastlerinnen und Bastlern? - Antwort auf Beck/Beck-Gernsheim" en: Zeitschrift für Soziologie, Año 22, Cuaderno 3, F. Enke Verlag, Stuttgart (p. 188-191).

Cárdenas, A. (2005): El sujeto y el proceso de transformación en las condiciones de trabajo en Chile: Elementos teóricos y empíricos para la discusión. Documento Electrónico, Oficina Internacional del Trabajo (OIT), Santiago de Chile.

Carpio, J. y Novacovsky, I. (2000): “Introducción”, en: Carpio, J. / Klein, E. / Novacovsky, I. (Eds.). Informalidad y exclusión social. Fondo de Cultura Económica/Siempro/ OIT, Argentina.

Castel, R. (1995): Les métamorphoses de la question sociale. Une chronique du salariat. Fayard.

CEPAL (1996): Quince años de desempeño Económico. América Latina y el Caribe, 19801995. Comisión Económica para América Latina y el Caribe, Santiago de Chile. 
CEPAL (2004): Panorama Social de América Latina. Comisión Económica para América Latina y el Caribe, Santiago de Chile.

Chacón, B. (1999): “Calidad del empleo y pobreza en Chile, 1990-1996”, en: R. Infante (Ed.), La calidad del empleo. La experiencia de los países latinoamericanos y de los Estados Unidos. OIT, Santiago de Chile.

Délano, B., Gálvez, T. y Todaro, R. (1989): Demandas de las mujeres asalariadas. Centro de Estudios de la Mujer (CEM), Santiago de Chile.

Díaz, X., Medel, J. y Schläen, N. (1996): Mujer, trabajo y familia: el trabajo a domicilio en Chile, Avances de Investigación, Centro de Estudios de la Mujer (CEM), Santiago de Chile.

Echeverría, M. y Uribe,V. (1998): “Condiciones de trabajo en sistemas de subcontratación”. Serie Documentos de Trabajo No81, OIT/ETM, Santiago de Chile.

Electoral (2005): Evolución inscripción de Jóvenes de 1988 al 2000, en: www.electoral.cl.

Espinosa M. y Morris P. (2002): "Calidad de vida en el trabajo. Percepciones de los trabajadores”. Departamento de Estudios, Cuadernos de investigación No16. Dirección del Trabajo Santiago de Chile.

Freud, S. (1959): Group Psychology and the Analysis of the Ego, Standard Edition, Norton.

García-Huidobro, G. (1999): "La capacidad generadora de empleo productivo de la economía chilena”, Serie de Reformas Económicas, N³1. Comisión Económica para América Latina (CEPAL), Santiago de Chile.

Geertz, Clifford (1973): The Interpretation of Cultures. New York: Basic Books.

Giddens, A. (1991): Selfidentity and Modernity. Polity Press, Londres.

Guerra, P. (1994): “El empleo precario y el empleo atípico. Revisión bibliográfica y propuestas para el debate", Serie Documentos de Trabajo N¹05. Programa de Economía del Trabajo (PET), Santiago de Chile.

Guzmán,V., Mauro,A. y Araujo K. (1999): Trayectorias laborales de mujeres. Cambios generacionales en el mercado del trabajo. Centro de Estudios de la Mujer (CEM), Santiago de Chile.

Guzmán V. y Mauro A. (2004): Las trayectorias laborales de mujeres de tres generaciones: coacción y autonomía en: Todaro R. y Yáñez S. (Eds.), El trabajo se transforma. Relaciones de producción y relaciones de género. Centro de Estudios de la Mujer (CEM), Santiago de Chile. 
Guzmán,V y Mauro A. (2004a): Trayectorias laborales masculinas y orden de género en: R. Todaro y S.Yáñez (Eds.), El trabajo se transforma. Relaciones de producción y relaciones de género. Centro de Estudios de la Mujer (CEM), Santiago de Chile.

Gubbins, V., Browne, F. y Bagnara, A. (2003): "Familia: innovaciones y desafios. Las familias chilenas en la década 1992-2002" en: INE, Cuánto y cómo cambiamos los chilenos. Balance de una década. Censos 1992-2002. Cuadernos Bicentenario, Santiago de Chile (p.191-249).

Hitzler, R. y Honer, A. (1994): “Über subjektive Konsequenzen der Individualisierung” en: Beck, U. y Beck-Gernsheim, E. (eds.), Riskante Freiheiten. Zur Individualisierung von Lebensformen in der Moderne. Suhrkamp, Frankfurt (p. 307-315).

Honneth,A. (1996): The Struggle for Recognition. MIT.

Huggins, M. (1991): Vigilantism and the State in Modern Latin America. Essays on Extralegal Violence. New York: Praeger Publishers.

INE (2003): Cuánto y cómo cambiamos los chilenos. Balance de una década. Censos 19922002. Cuadernos Bicentenario, Santiago de Chile.

INE (2005): Series estadísticas. Instituto Nacional de Estadísticas (INE) en: www.ine.cl.

Kohli, M. (1985): "Die Institutionalisierung des Lebenslaufs. Historische Befunde und theoretische Argumente" en: Kölner Zeitschrift für Soziologie. (p. 1-29)

Kohli, M. (1986): „Gesellschaftszeit und Lebenszeit. Der Lebenslauf im Strukturwandel der Moderne" en: Berger,J. (ed.), Soziale Welt, Sonderband 4, Schwartz, Göttingen.

- (1989). ,Institutionalisierung und individualisierung der Erwerbsbiographie. Aktuelle Veränderungstendenzen und ihre Folgen“ en: Brock, D., Leu, H.R. Preiß Vetter, H-R. (eds.), Subjektivität im gesellschaftlichen Wandel. DJI, München.

Koonings, K. (2004): Armed Actors. Organized Violence and State Failure in Latin America. London: Zen Books.

Kron, Th. (2000): "Individualisierung und soziologische Theorie-Einleitung", en: Kron,Th. (ed.), Individualisierung und soziologische Theorie. Lehrtexte Soziologie, Leske + Budrich, Opladen (p. 7-12).

Kumar, K. (1995): From Post Industrial to Post Modern societies. Blackwell.

Ley, K. (1984): “Von der Normal - zur Wahlbiographie?” en: M. Kohli/G. Robert (eds.): Biographie und soziale Wirklichkeit. Neue Beiträge undForschungsperspektiven. Metzler, Stuttgart (p. 239-260). 
Machado, L. (ed.) (2002): Qualitative Study of Schools with Outstading Results in Seven Latin American Countries. Santiago: Llece, UNESCO.

Martinez, J. y Palacios, M. (1995): Informe de la decencia. Santiago: SUR Editores.

Mauro A., Godoy L. y Guzmán V. (2001): Trabajo y relaciones de género: percepciones y prácticas de los varones. Centro de Estudios de la Mujer (CEM), Santiago de Chile.

MIDEPLAN (2004): Pobreza, distribución del ingreso e impacto distributivo del gasto social. Casen 2003,Vol. 1. Ministerio de Planificación y Cooperación (MIDEPLAN), Santiago de Chile.

MINEDUC (2001): Indicadores Estadísticos del Sistema Educativo del MERCOSUR 2001. Ministerio de Educación (MINEDUC) en: www.mineduc.cl.

MINEDUC (2002): Estadísticas de la Educación año 2001. Ministerio de Educación (MINEDUC).

MINEDUC (2005a): Resultados nacionales. Ministerio de Educación (MINEDUC), en: www.simce.cl.

MINEDUC (2005b): Reforma educacional Ministerio de Educación (MINEDUC), en: www.mineduc.cl.

Morgado Valenzuela, E. (1999): "Las Reformas laborales y su impacto en el funcionamiento del mercado del trabajo”, Serie Reformas Económicas, N`32. Comisión Económica para América Latina (CEPAL), Santiago de Chile.

OIT (1998): Chile: Crecimiento, empleo y el desafío de la justicia social. OIT, Santiago de Chile.

OIT (2003): Panorama laboral 2003. América Latina y el Caribe. OIT, Lima.

Oxhorn, P (2003): "Social Inequality, Civil Society and the Limits of Citizenship in Latin America", en: Eckstein, S. and Wickham-Crowley, T. (eds) What Justice? Whose Justice? Fighting for Fairness in Latin America Berkeley: University of California Press.

Palacios, M (2006): "Subjetividad y los límites del liberalismo en Chile", en Puertas Adentro: Masculino y Femenino en la Familia Contemporánea”, Valdés, X., CastelainMeunier y Palacios, M. Santiago: LOM-CEDEM, Santiago, Chile.

Palacios, M. y Martínez, J. (2005): “Conservadurism and Liberalism in Chile. Attitudes and Opiniones of Chilean Women at the end of the 20th Century". Journal of Latin American Studies, Cambridge University Press.Vol. 38, p. 1-34.

PNUD (2000): Desarrollo Humano en Chile. Más sociedad para gobernar el futuro. Programa de las Naciones Unidas para el Desarrollo, Santiago de Chile. 
PNUD (2002): Desarrollo Humano en Chile. Nosotros los chilenos: un desafí cultural. Programa de las Naciones Unidas para el Desarrollo, Santiago de Chile.

PREALC (1981): Sector informal. Funcionamiento y políticas, PREALC (Programa Regional del Empleo para América Latina y el Caribe) /OIT, Santiago de Chile.

Prebisch, R. (1950):"The economic development of Latin America and its principal problems" United Nations, New York.

Quijano, A. (1970): "Redefinición de la dependencia y proceso de marginalización en América Latina”, Ponencia para el curso de capacitación en planificación de los recursos humanos, ILPES/OIT, Santiago de Chile.

Raczynski,D. (1995) : "Focalización de programas sociales: lecciones de la experiencia de Chile" en: Pizarro, C., Raczynski, D. y Vial, J. (Eds.), "Políticas económicas y sociales en el Chile democrático", CIEPLAN, UNICEF, Santiago de Chile.

Reinecke, G. (1997): "Flexibilidad, innovaciones y cadenas productivas: La industria textil y del vestuario en Chile", Serie Documentos de Trabajo N55. OIT/ETM, Santiago de Chile.

Reinecke, G. (2004): La calidad del empleo, un enfoque de género. Taller Regional GPE Chile (material de presentación).

Tedesco, J.C. (1997): The New Educational Pact. Education, Competitiveness and Citizenship in Modern Society. Unesco.

Tedesco, J.C. (2003): "Los pilares de la educación del futuro" http://www.uoc.edu/ dt/20367/index/html

Tedesco,J.C. y López, N. (2002): Las condiciones de educabilidad de los niños y adolescentes de America Latina. Buenos Aires: IIPE.

Teitelboim, B. y Salfate, V. (2003): “Cambios sociodemográficos en educación" en: INE, Cuánto y cómo cambiamos los chilenos. Balance de una década. Censos 1992-2002. Cuadernos Bicentenario, Santiago de Chile (p.161-189).

Tokman,V. (2000):"El sector informal postreforma económica”, en: Carpio,J./ Klein, E./Novacovsky, I. (Eds.), Informalidad y exclusión social. Fondo de Cultura Económica - Siempro - OIT, Argentina.

Tokman,V. y Martínez, D. (1999): "Efectos de las reformas laborales: entre el empleo y la desprotección", en:Tokman,V./Martínez, D. (Eds.), Flexibilización en el margen: la reforma del contrato de trabajo. OIT, Lima (p. 11-38)

Turner, J. (1987): Social Theory Today. Stanford University Press.

Schroer, M. (2000):“Negative, positive und ambivalente Individualisierung - erwart- 
bare und überraschende Allianzen", en:Th. Kron (ed.), Individualisierung und soziologische Theorie. Lehrtexte Soziologie, Leske + Budrich, Opladen (p. 13-42).

Urmeneta, R. (1999): "La flexibilización de las relaciones de trabajo en Chile: contrataciones temporales, subcontratación y despidos", en:V. Tokman, V./D. Martínez (1999). Flexibilización en el margen: la reforma del contrato de trabajo. OIT, Lima.

Valdés, X, Castelain-Meunier y Palacios, M. (2006): "Puertas Adentro: Masculino y Femenino en la Familia Contemporánea”, Santiago: LOM-CEDEM, Santiago, Chile.

Valdés, X (2007): Notas sobre la metamorfosis de la familia en Chile. CEPAL.

Winnicott, D. (1993): Los Procesos de Maduración y el Ambiente Facilitador, Paidós.

Wormald, G. y Ruiz-Tagle, J. (1999): “Exclusión social en el mercado del trabajo. El caso de Chile". Serie Documentos de Trabajo N¹06. OIT/Fundación Ford, Santiago de Chile. 